\title{
COMPARISON OF QUICK'S, OWREN'S, AND WARE'S TECHNIQUES FOR THE CONTROL OF ANTI- COAGULANT THERAPY
}

BY

\author{
M. TOOHEY \\ From the Anticoagulant Unit, New End Hospital, London, N.W.3
}

(RECEIVED FOR PUBLICATION OCTOBER 31, 1957)

Anticoagulant therapy is now in widespread use and is generally accepted as being of real value in the treatment of myocardial infarction and other thrombo-embolic diseases. In the last few years long-term continuous treatment with anticoagulants to prevent further attacks of infarction has been more widely adopted. With appreciable numbers of patients, both in-patients and outpatients, receiving anticoagulant drugs, it is perhaps timely to evaluate the different methods used for the control of anticoagulant therapy.

Until fairly recently Quick's method was almost universally employed and considered as the simplest and best means of achieving laboratory control of anticoagulant therapy. Even to-day it is still the method most widely used. More recently, however, Owren's " $\mathrm{P}$ and $\mathrm{P}$ " (prothrombin and proconvertin) technique (Owren and Aas, 1951) has been advocated as being more sensitive and reliable, and it is claimed that with its use there is a lower incidence of haemorrhagic manifestations. Ware and Stragnell (1952), using a modified version of Owren's technique, found that it gave better results than Quick's method.

In the New End Hospital anticoagulant unit, Quick's, Owren's, and Ware's methods were used simultaneously to control anticoagulant therapy of in-patients over a period of several months, and in all 800 blood samples were tested by all three techniques. It was found that, apart from the method of preparation of the prothrombin- and proconvertin (factor VII)-free ox plasma, Owren's method was better than Ware's.

When performing the test by Ware's method, the test plasma is diluted with water and only half as much calcium and thromboplastin is added as is used of the other reagents. As a result the difference between the minimum and maximum therapeutic clotting times is much smaller than with Owren's method. In practice, however, this shorter time is less convenient, especially when large numbers of tests are being made. Again, ू below a level of $10 \%$ there was sometimes a 을 marked lack of correlation between the two methods with, on the whole, more reliable results with Owren's technique.

After this preliminary trial with all three methods, a further comparison was then made between Quick's and Owren's methods. In all, 2,100 blood samples were tested with both tech- 9 niques over a period of 16 months for the control of in-patients on anticoagulant treatment. As a result of this trial there appeared to be no doubt that Owren's method had one decided advantage, i.e., it reflected changes in the "prothrombin" level ("prothrombin" being taken as meaning both prothrombin and proconvertin, both of which are affected by coumarin drugs) 24 , or even more, hours earlier than did Quick's method. Quick's method seemed to lag behind Owren's. The great advantage ? with Owren's method was therefore that one was able to make the necessary adjustments in the dosage of the anticoagulant drug more quickly and easily. This meant that the correct maintenance dose of the anticoagulant was more quickly arrived at and lessened the number of $\frac{D}{O}$ blood tests required for control of the treatment.

For treatment of an acute illness it is necessary $N$ to bring the patient under therapeutic control as quickly as possible. In these circumstances, when $\mathrm{N}^{-}$ frequent and big changes in the dose of the anti- $\omega$ coagulant drug are usually necessary, and when it is possible to take fairly frequent blood tests, Owren's method does enable one to adjust the dose more rapidly and easily. One point must, however, be emphasized. It has been said that because Owren's method is more sensitive than Quick's it $\vec{D}$ enables a more accurate estimate to be made of $\frac{?}{\mathbb{Q}}$ the dose of the anticoagulant drug required. This $\varnothing$ is not strictly true. Quick's test is just as "sensi- ? tive" to the effects of small changes in the dosage 8 
of anticoagulant drugs, but it takes longer for these effects to be noticeable. Therefore, with longterm anticoagulant therapy, when blood tests are made every few weeks, or even at longer intervals, it is difficult to see how Owren's technique will materially improve on Quick's method. Indeed, in a busy out-patient clinic, where large numbers of tests have to be made quickly to avoid keeping the patients waiting too long, the speed and simplicity of Quick's method are a decided advantage. Experience with Owren's method for out-patient therapy in this unit is, however, too limited for a definite conclusion yet to be drawn.

\section{Preparation of Reagents for Owren's Method}

For successful results with Owren's technique it is essential to prepare a thromboplastin with a constant and reproducible activity and a reliable prothrombin and proconvertin-free ox plasma. Unless these two reagents give constant reproducible clotting times, the results with Owren's method will be most unsatisfactory. Only familiarity with the preparation of these reagents will give the desired results, but the methods of preparation outlined below have been found to be reliable. In the main they are very similar to Owren's instructions, but minor details are worth emphasizing.

Thromboplastin. - As advocated by Owren and myself for many years, for Quick's technique a saline extraction of human brain is used. For Quick's test I have always felt that a saline extraction of brain gives better and more reproducible results than an acetone-dried brain extract. Owren for his technique says, "A thromboplastin with a constant and reproducible activity is not obtained by extraction of dried brain substance." With this I fully agree. It must be stressed that for Owren's method, as for Quick's, the first essential for reliable results is a potent thromboplastin which gives constant reproducible activity. The following method is used to prepare the thromboplastin:

A whole human brain is used. It is washed under tap water and freed of membranes and blood vessels. Any brain tissue with obvious blood is discarded. The brain is then emulsified by a mechanical homogenizer for about 60 seconds to produce a fine emulsion. For this emulsification $2 \frac{1}{2}$ to 3 litres (Owren uses $1 \frac{1}{2}$ litres) of normal saline $(0.9 \mathrm{~g}$. sodium chloride per $100 \mathrm{ml}$. distilled water) heated to $45^{\circ} \mathrm{C}$. to $50^{\circ} \mathrm{C}$. is used. This fine emulsion is next centrifuged for 15 minutes only at 2,000 r.p.m. to remove all the coarser sediment. The supernatant fluid is poured off ; it does not matter at this stage if a little sediment of brain tissue is decanted over into the supernatant fluid. The supernatant extract is incubated at $37^{\circ} \mathrm{C}$. for two hours, no longer. During the period of incubation the brain extract should be thoroughly shaken for several minutes on three or four occasions.

After two hours' incubation the brain extract is centrifuged for a second time at 2,000 r.p.m. for about 18 to 20 minutes. After centrifuging it will be found that there is a small sediment of brain tissue present. The supernatant fluid is very carefully poured off, making sure this time that no sediment is decanted over. This point is essential, as in the final brain extract there must be no sediment, visible to the naked eye, unless the extract has been standing for many hours. This final brain extract is now ready to be standardized and tested for activity. It must give a Quick prothrombin time of 11 to 13 seconds, ideally 12 seconds, with pooled normal control plasma. If the brain is too concentrated it may have to be diluted, as Owren points out, to optimum strength. This means the least concentrated brain extract which will give a Quick time of 11 to 13 seconds. The following procedure for standardization has been found to give reliable and reproducible results.

After the incubated extract has been centrifuged and the supernatant fluid decanted, great care being taken not to allow any sediment to spill over, a colorimetric reading is taken. With a red filter (640 wavelength) in a $15 \mathrm{~mm}$. tube (an E.E.L. colorimeter giving a reading of 41 with the E.E.L. permanent opalescent standard is used here) the colorimetric reading should be in the region of 20 to 30 . If the reading is below 18 (which is unlikely unless the brain extract has been centrifuged for much longer times than stated above) resuspend the supernatant in the sediment and centrifuge again but this time for a shorter period, say 15 minutes at 2,000 r.p.m., instead of 20 minutes. If the colorimetric reading is between 20 and 30 , the $p \mathrm{H}$ of the supernatant extract is adjusted to 7.35 with $\mathrm{N} / 2$ caustic soda. After adjusting the $p \mathrm{H}$ add one-tenth the volume of Owren's buffer (see below).

A Quick time is now estimated with this extract with normal control plasma and the time should be between 11 and 13 seconds. If the Quick time is above 14 seconds it is advisable to prepare another brain extract. If the Quick time is between 11 and 13 seconds the brain extract is now diluted with buffersaline, as necessary, to give a colorimeter reading of 18 to 20 . (Buffer-saline is made up by adding one volume of Owren's buffer to nine volumes of normal saline.) The brain extract is stored at $-20^{\circ} \mathrm{C}$. in tightly stoppered tubes. Tubes ( 3 in. by $\frac{1}{2}$ in.) are convenient to use as they can be put directly into the water-bath to warm up rapidly to the desired temperature of $37^{\circ} \mathrm{C}$.

When a fresh brain extract is made up for use. it is perhaps desirable (although not essential for Owren's method) to compare it with the brain extract in current use for control of anticoagulant therapy. Therefore, in addition to making sure that the normal control value is correct, i.e., between 11 and 13 seconds, the plasma of a patient on anticoagulant therapy with a prolonged Quick time should be tested 
with the old and new brain extracts, and with both extracts the Quick time should be approximately the same to within a few seconds.

With experience in estimating the optimum concentration of brain extract, the above procedure has given brain extracts yielding constant and reproducible results over the last 18 months. One quickly learns to gauge the correct centrifuging times so as to avoid getting too concentrated or too dilute a brain extract. For Owren's method it is essential not to have too concentrated a brain extract and yet it must be sufficiently potent to give constant and reproducible results.

It is perhaps as well to note the difference between the procedure outlined here for preparing the brain extract and that advised by Owren. These differences are mainly a matter of convenience. Owren uses $1 \frac{1}{2}$ litres of normal saline for emulsifying a whole brain, whereas here it has been found much more convenient to use $2 \frac{1}{2}$ litres, as less dilution is then necessary at a later stage in the preparation. Again Owren, after emulsification of the brain, allows the emulsified brain to stand at room temperature until the temperature of the extract falls from $50^{\circ} \mathrm{C}$. to $20^{\circ} \mathrm{C}$. This may, however, take the best part of the day. By doing an initial short centrifuging for about 15 minutes at 2.000 r.p.m. and incubating the supernatant fluid for two hours, it has been found possible to speed up the extraction and still get a sufficiently potent thromboplastin. Finally, instead of adding by trial and error different amounts of buffer-saline to arrive at the optimum concentration, the colorimetric readings quoted above give a very accurate guide to the optimum concentration of the final extract and result in an easily standardized thromboplastin. It is well worth while taking considerable trouble in preparing the brain extracts so as to get as potent and standardized a thromboplastin as possible.

Prothrombin and Proconvertin-free Ox Plasma.Ware's method for preparation of the prothrombinand proconvertin-free ox plasma has been found to be the simplest and most reliable. The following technique is used here:

Bovine blood is collected in wide-mouthed jars containing $100 \mathrm{ml}$. of $2 \%$ potassium oxalate for $900 \mathrm{ml}$. of blood. In collecting the blood it is important to mix well to prevent fine clots. To separate the plasma it is necessary to centrifuge for 30 to 45 minutes at 2.000 r.p.m. Adsorption of the ox plasma is carried out using barium sulphate, 20 to $25 \mathrm{~g}$. for each litre of plasma. (Most of the ordinary commercial preparations of barium sulphate as used for $x$-ray examinations give reliable results.) Adsorption is carried out for 20 to 30 minutes at room temperature, and about every four to five minutes the plasma must be thoroughly shaken to disperse the barium sulphate throughout the plasma. The plasma is then centrifuged for 30 minutes at 2,000 r.p.m. to remove the barium sulphate. The plasma is next filtered once through a Seitz filter, using a $50 \%$ asbestos filter pad.
One $14 \mathrm{~cm}$. pad is used for each $400 \mathrm{ml}$. of plasma. As the filtration is very slow, pressure is necessary, usually 10 to $15 \mathrm{lb}$. per sq. inch.

The plasma is now tested by doing a Quick time. which should be longer than 20 minutes. An additional method of testing to make sure that sufficient prothrombin and proconvertin have been removed is to add an equal quantity of normal serum to the plasma and then do a Quick time, which should be 80 seconds or more.

Finally, the $p \mathbf{H}$ of the plasma is adjusted to 7.35 by adding $\mathrm{N} / 2$ hydrochloric acid, and the plasma is stored in tightly stoppered tubes at $-20^{\circ} \mathrm{C}$., where it keeps stable indefinitely.

For the collection of bovine blood it is important to note that potassium oxalate $(2 \%$ or $2.5 \%)$ must be used and not citrate, because if citrate is used it is not possible to get a sufficiently prothrombin-free ox plasma by adsorption with barium sulphate. Once the plasma has been thawed out it must not be refrozen. The plasma remains stable at room temperature for four to six hours at least. and probably for most of the day.

Owren. in preparing the ox plasma, uses citrated blood and filters through a $20 \%$ asbestos filter pad. following this with a second filtration through $50^{\circ}$. asbestos. Here it has been found that with this technique the speed of filtration and the amounts of plasma filtered through each filter pad require constant attention. much more so than with Ware's method. Using Ware's technique invariably a plasma sufficiently free of prothrombin and proconvertin is obtained. whilst at the same time it is much easier and quicker to carry out.

Owren gives as an alternative method adsorption with barium sulphate alone, using $100 \mathrm{~g}$. for each litre of plasma and adsorbing for five minutes. One adsorption with barium sulphate, even using such large amounts, does not always give the desired results and it is often necessary to repeat the adsorption using fresh barium sulphate after removing the first lot of barium by centrifuging for 30 minutes at 2.000 r.p.m. As Ware's technique invariably gives the desired result, as well as being quicker and easier to carry out, here it is always used. It also has the advantage that filtration through a $50 \%$ asbestos pad will remove fine particles of barium sulphate that have not been removed by centrifuging. It should be noted that, whereas Ware uses a $40 \%$ asbestos filter, a filter pad of $50^{\circ} \%$ asbestos content is recommended here.

Pooled Normal Human Plasma.-To prepare the standard dilution curve and also for checking each day`s routine tests. plasma is collected from 10 to 12 normal people. The pooled plasma is stored in small amounts at $-20^{\circ} \mathrm{C}$. in tightly stoppered tubes. The bulk of the plasma is put up in accurately measured $0.5 \mathrm{ml}$. amounts suitable for each day's routine tests. A few tubes with $1.5 \mathrm{ml}$. amounts are stored for preparing the standard curve. There is a very slight diminution in the prothrombin and proconvertin con- 
tent of the stored pooled normal plasma over several months, but not sufficient to interfere with the normal routine testing.

Citrate-heparin Anticoagulant.-It is a common finding that, when citrate or oxalate is used for collection of blood specimens, there is often an appreciable shortening of the Quick time after the blood has been standing for a short time. This shortening of the Quick time is supposed to be due to activation of proconvertin: the change is entirely unpredictable and may be very slight or considerable. In 18 to 24 hours the Quick time usually returns to its original value.

Ware devised a special anticoagulant which eliminates this activation of proconvertin. He adds $100 \mu \mathrm{g}$. of standard heparin to each millilitre of anticoagulant used, whether oxalate or citrate.

For collection of the blood samples Owren (Owren, 1956) advises against the use of oxalate, as he has found that the calcium-oxalate precipitate adsorbs a small amount of prothrombin and proconvertin. This finding can be confirmed, and here it has been found that the citrate-heparin anticoagulant is preferable to oxalate-heparin, so $3.13 \%$ sodium citrate is used.

Owren's Buffer.-This is used for preparation of the thromboplastin and also for making up the diluting fluids for carrying out the actual tests. Sodium diaethyl barbiturate, $11.75 \mathrm{~g}$., sodium chloride, 14.67 g., and $0.1 \mathrm{~N}$. hydrochloric acid, $430 \mathrm{ml}$., are mixed together: distilled water is then added to bring the total volume up to $2,000 \mathrm{ml}$. This buffer should have a $p \mathrm{H}$ of 7.35 . It is best stored at $0.5^{\circ} \mathrm{C}$. to prevent the growth of moulds.

\section{Preparation of the Standard Dilution Curve}

Whenever a fresh batch of reagent, such as the thromboplastin, ox plasma. or fresh pooled control human plasma, is used a new standard curve must be prepared. The more carefully the thromboplastin extracts are standardized by arriving at the optimum concentration of the particle size of brain tissue in the extract, the smaller will be the difference between the standard curves prepared with different brain extracts. For convenience' sake attempts are made to standardize each fresh batch of brain extract with the brain extract last used. Nevertheless, no matter how much care is taken to do this, minor differences will appear with each new batch of brain extract and ox plasma, so that new standard dilution curves must be prepared for each fresh reagent.

Owren strongly advises that the concentration of heparin, citrate, ionic strength, and $p H$ should be kept constant in all dilutions made when preparing the standard dilution curve. To do this two dilutions of human plasma are necessary. First, a primary dilution using what he calls diluting fluid $I$, which is made up by mixing $100 \mathrm{ml}$. of citrate-heparin anticoagulant and $500 \mathrm{ml}$. of normal saline. This diluting fluid has the same citrate, heparin, and ionic strength as the normal or test plasma collected in the special citrate-heparin anticoagulant, using 1 vol. of anticoagakant to 9 vols. of blood.
Using diluting fluid I, a primary dilution of the normal pooled control human plasma (collected and stored as mentioned earlier) is made to give $50 \%$, $25 \%, 12.5 \%$, and $6.25 \%$ concentrations of plasma. Each of these dilutions has the same concentration of citrate and heparin and the same ionic strength as the original $100 \%$ concentration of plasma. A second dilution is now made from each of the $100 \%, 50 \%$, $25 \%, 12.3 \%$, and $6.25 \%$ concentrations, but this time a second diluting fluid is used. Owren uses a second diluting fluid because he has found that his method works better when the final plasma dilution has a lower concentration of citrate and heparin than the original specimen of plasma. Owren's diluting fluid II, as he calls it, is made up as follows: $\operatorname{mix} 200 \mathrm{ml}$. of Owren's buffer, $200 \mathrm{ml}$. of $0.754 \mathrm{~g}$. sodium citrate per $100 \mathrm{ml}$., and $600 \mathrm{ml}$. of normal saline. In practice it is even more convenient to use a still lower concentration of citrate and heparin in the final dilutions. and excellent results have been obtained by using as a second diluting fluid $200 \mathrm{ml}$. of Owren's buffer and $800 \mathrm{ml}$. of $1 / 5$ normal saline $(0.18 \mathrm{~g}$. sodium chloride per $100 \mathrm{ml}$. distilled water).

To prepare the second dilutions, a 1 in 10 dilution is made from each of the $100 \%, 50 \%, 25 \%, 12.5 \%$. and $6.25 \%$ concentrations made with diluting fluid $I$. To do this $0.2 \mathrm{ml}$. is pipetted from each of these dilutions into $1.8 \mathrm{ml}$. of the second diluting fluid. Each of these dilutions is then tested by Owren's method and the clotting times plotted on double logarithmic paper: a straight line should be obtained. Owren states that the line tends to "bend upwards" below $5 \%$. It has been found here that no matter how much care is taken in preparing the standard dilution curve the line tends to curve upwards at about $7 \%$ or $8 \%$. In practice this is of little significance.

It should be noted that with Owren's method of preparing the standard dilution curve the $100 \%$ value for normal plasma corresponds to a final dilution of $10 \%$, the $50 \%$ and $25 \%$ concentrations correspond respectively to final dilutions of $5 \%$ and $2.5 \%$, and so on. This is because with Owren's technique all the actual blood tests are carried out in a dilution of 1 in 10. This method of preparing the standard dilution curve sounds complicated, but with practice it becomes quite simple. All dilutions must be made with great care.

In a fairly busy anticoagulant unit enough brain extract and ox plasma can easily be prepared at one time to last about three to four months; it is thus necessary to prepare a standard dilution curve only once in this period. During the 18 months in which Owren's method has been in routine use in this anticoagulant unit many different brain extracts and ox plasmas have been prepared, and the standard dilu. tion curves do not differ markedly from each other. The $50 \%$ dilution of pooled normal plasma usually gives a clotting time of between 35 and 40 seconds with different batches of reagents; the $25 \%$ dilution of pooled normal plasma usually gives a clotting time 
in the region of 53 to 58 seconds and the $12.5 \%$ dilution times of 82 to 94 seconds.

\section{Routine Tests}

Before each day's tests of the blood specimens a control test is done to make sure that all reagents have been properly standardized. The $25 \%$ dilution of the normal pooled plasma is the most suitable for test purposes. For convenience and speed the bulk of the pooled normal human plasma is stored in accurately measured $0.5 \mathrm{ml}$. amounts in tubes at $-20^{\circ} \mathrm{C}$. The plasma is thawed rapidly in a water-bath at $37^{\circ} \mathrm{C}$. and then $1.5 \mathrm{ml}$. of diluting fluid $\mathrm{I}$ is added and carefully mixed. This gives a primary dilution of $25^{\circ}$. Then $0.2 \mathrm{ml}$. of this dilution is added to $1.8 \mathrm{ml}$. of the second diluting fluid and Owren's test is carried out in duplicate. The clotting times should correspond, within a few seconds, to the times of the $25^{\circ} \%$ concentration on the standard dilution curve.

Having carefully checked the reagents, each plasma is tested in duplicate by Owren's method and the actual clotting times in seconds converted to a percentage from the standard dilution curve. The actual tests are carried out as follows: Thromboplastin, $0.2 \mathrm{ml}$., $0.2 \mathrm{ml}$. of ox plasma, and $0.2 \mathrm{ml}$. of test plasma diluted 1 in 10 with diluting fluid II are mixed together and warmed in a water-bath at $37^{\circ} \mathrm{C}$. for at least two to three minutes. Owren's test. like Quick's, or even more so, is very sensitive to minor variations in temperature. It is therefore very important for all reagents and the diluted test plasma to be kept at $37^{\circ} \mathrm{C}$. for several minutes. Calcium chloride, $0.2 \mathrm{ml}$.. previously warmed to $37^{\circ} \mathrm{C}$., is then added and the clotting time measured. Owren says that calcium chloride of optimum concentration should be added and for each fresh batch of reagents the optimum concentration of calcium chloride must be ascertained. In practice $\mathrm{M} / 30$ calcium chloride invariably gives sufficiently accurate results and therefore this strength is always used.

\section{Collection of Blood Specimens}

As with all blood clotting studies, it is essential that all the blood specimens should be taken with a minimum of trauma and as quickly as possible. The needle must always be removed from the syringe and the blood at once intimately mixed with the citrateheparin anticoagulant. All the test plasmas should be carefully examined before testing for the presence of fine clots which would give false results.

For the control of anticoagulant therapy Owren advises a " $\mathbf{P}$ and $\mathbf{P}$ " level of $10^{\circ}$, to $30^{\circ} \%$. I believe that a lower level is necessary for treatment of acute thrombo-embolic diseases, and I try to maintain all patients at about $10 \%$ to $14 \%$. Levels between $6 \%$ and $8 \%$ should be avoided for any period of time and levels below $5^{\circ} \%$ are hazardous.

\section{Summary and Conclusions}

The results of controlling anticoagulant therapy by the simultaneous use of Quick`s, Owren's, and
Ware's modification of Owren's technique have $\ddot{\Rightarrow}$ been compared. All three methods were carried $\stackrel{?}{?}$ out on 800 test plasmas, and Owren's method was found to be more convenient and slightly better than Ware's. Quick's and Owren's methods were then further compared and, in all, 2,100 test $\Omega$ plasmas from in-patients on anticoagulant therapy were tested by both methods over a period of $16 \vec{\circ}$ months. There appeared to be no doubt that Owren's technique gave better results for the con- $\vec{\omega}$ trol of anticoagulant therapy, at least for in- $\frac{\Omega}{2}$ patient, as opposed to out-patient, long-term ? therapy. Owren's method reflects changes in the $\overrightarrow{-}$ blood 24 or even more hours before Quick's $\overrightarrow{-}$

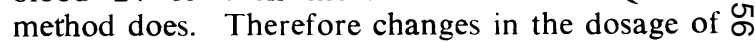
the anticoagulant drug can be made more rapidly 음 and the correct maintenance dose thus more quickly determined. In the long run Quick's פ method will reflect even the most minute change in the dosage of the anticoagulant drug, but it takes longer to do so. For anticoagulant therapy for an acute thrombo-embolic episode, when it is essen- o tial to get and maintain the patient under adequate ${ }^{\infty}$ anticoagulant cover as rapidly as possible and where it is usually necessary to make rapid and big changes in the dose of the anticoagulant drug, Owren's method is definitely better than Quick's. ̊ For out-patient long-term therapy, however. $\stackrel{\AA}{\varrho}$ where the question of urgency does not arise and $\overrightarrow{\overrightarrow{0}}$ where blood specimens are taken at intervals of 3 several weeks or more, I have yet to be convinced that Owren's method is superior to Quick's method.

For Owren's method it is essential to prepare an active and potent thromboplastin giving constant and reproducible results. The concentration of the $\frac{3}{3}$ thromboplastin is critical, and both a too concentrated and a too dilute thromboplastin must be $\frac{0}{3}$ avoided. The method outlined above for prepara- 0 tion of a saline extract of human brain has given very reliable and constant results with Owren's $\frac{7}{0}$ technique (and also with Quick`s method). A saline extract of human brain keeps stable $N$ indefinitely at $-20^{\circ} \mathrm{C}$. An acetone-dried extract, $N$ besides being more troublesome to prepare, does N not give such constant results.

For preparation of the prothrombin- and proconvertin-free ox plasma Ware's technique, using adsorption with barium sulphate followed by filtration through a $50 \%$ asbestos filter pad, gave better results than Owren's and it is also much quicker and simpler to carry out.

Better results were obtained by using a lower $\frac{?}{\mathbb{D}}$ concentration of citrate and heparin and a lower $\varrho$ ionic strength for dilutions of the test plasma than advised by Owren. The addition of heparin to the 
anticoagulant for collection of the blood specimens does, as pointed out by Ware, stabilize the clotting factors for several hours at least, presumably by preventing the activation of proconvertin. As observed by Owren, the calciumoxalate precipitate does adsorb small amounts of prothrombin and proconvertin, so it is best to use citrate-heparin and not oxalate-heparin to collect the blood specimens.

In carrying out the actual test the diluted test plasma, thromboplastin and ox plasma must be warmed together for at least two to three minutes at $37^{\circ} \mathrm{C}$, as minor variations in temperature can have significant effects.

The thromboplastin, ox plasma, and pooled human control plasma all keep stable for many months, at least three to four, when stored at $-20^{\circ} \mathrm{C}$. in tightly stoppered tubes. The thromboplastin and ox plasma remain active and stable at room temperature for four to six hours.

\section{REFERENCES}

Owren, P. A. (1956). Personal communication.

- and Aas, K. (1951). Scand. J. clin. Lab. Invest., 3, 201.

Ware, A. G., and Stragnell, R. (1952). Amer. J. clin. Path., 22, 791. 